\title{
Genetic associations with neuroendocrine tumor risk: results from a genome-wide association study
}

\author{
Yeting Du1, Monica Ter-Minassian1,3, Lauren Brais³, Nichole Brooks³, \\ Amanda Waldron'3, Jennifer A Chan'3, Xihong Lin', Peter Kraft', \\ David C Christiani1,2 and Matthew H Kulke ${ }^{3}$
}

1 Harvard School of Public Health, Boston, Massachusetts, USA

2Massachusetts General Hospital, Boston, Massachusetts, USA

3Dana-Farber Cancer Institute, Boston, Massachusetts, USA
Correspondence should be addressed to $\mathrm{M} \mathrm{H}$ Kulke Email

matthew_kulke@dfci. harvard.edu

\begin{abstract}
The etiology of neuroendocrine tumors remains poorly defined. Although neuroendocrine tumors are in some cases associated with inherited genetic syndromes, such syndromes are rare. The majority of neuroendocrine tumors are thought to be sporadic. We performed a genome-wide association study (GWAS) to identify potential genetic risk factors for sporadic neuroendocrine tumors. Using germline DNA from blood specimens, we genotyped 909,622 SNPs using the Affymetrix 6.0 GeneChip, in a cohort comprising 832 neuroendocrine tumor cases from Dana-Farber Cancer Institute and Massachusetts General Hospital and 4542 controls from the Harvard School of Public Health. An additional 241 controls from Dana-Farber Cancer Institute were used for quality control. We assessed risk associations in the overall cohort, and in neuroendocrine tumor subgroups. We identified no potential risk associations in the cohort overall. In the small intestine neuroendocrine tumor subgroup, comprising 293 cases, we identified risk associations with three SNPs on chromosome 12, all in strong LD. The three SNPs are located upstream of $E L K 3$, a transcription factor implicated in angiogenesis. We did not identify clear risk associations in the bronchial or pancreatic neuroendocrine subgroups. This large-scale study provides initial evidence that presumed sporadic small intestine neuroendocrine tumors may have a genetic etiology. Our results provide a basis for further exploring the role of genes implicated in this analysis, and for replication studies to confirm the observed associations. Additional studies to evaluate potential genetic risk factors for sporadic pancreatic and bronchial neuroendocrine tumors are warranted.
\end{abstract}

Endocrine-Related Cancer (2016) 23, 587-594

\section{Introduction}

The current annual incidence of neuroendocrine tumors in the United States is estimated to be 5.25 per 100,000 population, and the prevalence is estimated to exceed 100,000 individuals (Yao et al. 2008).
Neuroendocrine tumors are characterized by welldifferentiated morphologic features and the ability to secrete neuropeptides, resulting in characteristic clinical syndromes. The best known of these syndromes

Published by Bioscientifica Ltd 
is the carcinoid syndrome, which is characterized by excess secretion of serotonin and the development of flushing, diarrhea and right-sided valvular heart disease (Kulke \& Mayer 1999). Neuroendocrine tumors arising in the pancreas are categorized as 'pancreatic neuroendocrine tumors' or 'islet cell tumors' and comprise approximately $10 \%$ of neuroendocrine tumors (Yao et al. 2008). Neuroendocrine tumors arising in other anatomic locations are generally described as 'carcinoid tumors' (Kulke \& Mayer 1999). The most common sites for carcinoid tumors are the lungs and bronchi, which account for up to $30 \%$ of all neuroendocrine tumors, with small intestine, appendix and rectal carcinoid tumors comprising the majority of the remaining sites (Yao et al. 2008). Within the gastrointestinal tract, carcinoid tumors arising in the small intestine are the most common (Kulke 2007).

Mendelian syndromes associated with the development of neuroendocrine tumors have been well described. Multiple endocrine neoplasia type 1, caused by mutations in the tumor suppressor gene MEN1, has been associated with the development of neuroendocrine tumors of the pancreas, lung and thymus, as well as adenomas of the parathyroid and pituitary glands. Additional modifying genetic factors also appear to play a role in this condition (Thevenon et al. 2015). Multiple endocrine neoplasia type 2 (MEN2), caused by mutations in the RET proto-oncogene, is characterized by the development of paraganglioma/pheochromocytoma and medullary thyroid cancer. Pheochromocytoma/ paraganglioma, a rare group of neuroendocrine tumors not specifically included in our study, are commonly associated with germline mutations, which have been described in a number of genes, including VHL, NF1, $R E T$, genes in the succinate dehydrogenase pathway, and, less commonly, TMEM127, MAX, EPAS1, FH and MDH2 (Fishbein 2016).

Inherited syndromes, however, are thought to account for only a small minority of pancreatic neuroendocrine and carcinoid tumor cases (Leotlela et al. 2003). Furthermore, no clearly established environmental risk factors for neuroendocrine tumors have been identified. Case control studies have provided conflicting data regarding potential links between the development of neuroendocrine tumors and smoking or other exposures (Hassan et al. 2008a,b, Rinzivillo et al. 2015). An inherited basis for presumed "sporadic" neuroendocrine tumors has been suggested by evidence of familial clustering of neuroendocrine tumors described in both case series and population-based studies. An institutional analysis of 243 carcinoid cases, as well as an initial populationbased analysis of a Swedish Family-Cancer Database comprising 10.2 million individuals, identified increased incidence rates of carcinoid tumors in first-degree relatives (Babovic-Vuksanovic et al. 1999, Hemminki \& Li 2001). A subsequent, more recent analysis of the Swedish database confirmed the increased risk in first-degree relatives of individuals with carcinoid tumors, reporting a relative risk of 4.33 (Hiripi et al. 2009). Another study, combining family cancer datasets from Sweden and Finland, provided specific evidence of familial associations in individuals with small intestine carcinoid tumors: siblings of individuals with small intestine carcinoid tumors had a 30-fold higher risk of developing the same condition, and parents or children a 10-fold increased risk (Kharazmi et al. 2013). A recent study that focused on families with small intestine carcinoid tumors revealed the presence of multifocal and independent tumors arising within the small intestine, consistent with the presence of an inherited susceptibility gene (Sei et al. 2016). Inherited mutations in the gene IPMK were identified in one such family, though not in other families (Sei et al. 2015).

Initial attempts at performing genome-wide association studies to identify genetic risk factors in neuroendocrine tumors have been limited by small sample size. Although candidate loci have been reported in these studies, they have not been replicated and the results of these initial studies have been inconclusive (Ter-Minassian et al. 2011, Walsh et al. 2011). To further investigate a potential heritable basis for sporadic neuroendocrine tumors, we performed a large-scale genome-wide association study evaluating over 900,000 SNPs, using the Affymetrix 6.0 Chip. We genotyped 832 specimens from a prospectively enrolled population of neuroendocrine tumor patients from our center, and compared genotype frequencies to over 4500 control cases from two large cohort studies, the Nurse's Health Study and the Health Professionals Follow-Up Study. We analyzed potential risk associations in the cohort overall and within NET subgroups.

\section{Methods}

\section{Study population}

Patients with a confirmed diagnosis of NET (excluding small cell lung cancer) were recruited to an Institutional Review Board-approved study in the gastrointestinal clinic at Dana-Farber Cancer Institute (DFCI). A total of 1396 patients with neuroendocrine tumor were recruited

Published by Bioscientifica Ltd 
to a prospective follow-up study between 2003 and 2012 . Of these patients, 1265 provided blood specimens, and 942 specimens were subsequently genotyped. Twentyfive of these cases had previously also been evaluated in the previously described multicenter study of small intestine neuroendocrine tumor risk, and 370 of the cases had been included in the prior study from our center evaluating potential risk associations in candidate genes (Ter-Minassian et al. 2011, Walsh et al. 2011). Consent had been obtained from each patient after a full explanation of the purpose and nature of all procedures used. Baseline clinical and demographic information was derived from both questionnaires and from the medical record. All pathology was reviewed in the Pathology Department at Dana-Farber/Brigham and Women's Cancer Center at the time of patient consultation.

Due to a limited number of bronchial neuroendocrine tumors in the Dana-Farber cohort, an additional set of 75 patients with histologically confirmed welldifferentiated (typical or atypical) neuroendocrine tumor were included from Harvard Lung Cancer Susceptibility Study conducted from 1992 to 2011 at the Massachusetts General Hospital/Harvard Cancer Center, also in Boston. This study was approved by the Human Subjects Committees of Massachusetts General Hospital and the Harvard School of Public Health in Boston, MA, USA. Patients completed a questionnaire before time of first visit that included clinical and demographic data, as well as past medical and family cancer history. Unreported clinical information, including tumor type, histology and stage was extracted from the medical record.

Two hundred and ninety-one healthy controls were recruited from Dana-Farber Cancer Institute (DFCI) and genotyped along with the cases for quality control (QC). Healthy controls were recruited from friends and nonblood-related family members who accompanied the patients to the Dana-Farber Cancer Institute clinic from and volunteered to participate in the study. These DFCI controls completed the same questionnaire as the cases. The primary comparator controls consisted of 4622 healthy individuals identified from the Nurses Health Study (NHS) and the Health Professionals Follow-Up Study (HPFS). These controls are henceforth referred to as Harvard controls.

\section{Genotyping and quality control}

DNA samples for cases and for the DFCI controls (used for QC) were genotyped using the Affymetrix GeneChip Human Mapping 6.0 set (Affymetrix) at the Broad
Institute, Cambridge, MA. The Harvard controls had been previously genotyped on the Affymetrix 6.0 platform. We genotyped 909,622 SNPs with 1308 total samples (this does not include 14 HapMap samples genotyped making the total 1322). In brief, before merging, an initial Affymetrix quality control check identified 35 samples that did not pass the FQC genotyping call rate of $>86 \%$ on a subset of 3022 SNPs or that failed a process QC. SNPs were identified on the remaining 1273 samples using the Birdsuite calling algorithm (Korn et al. 2008). Standard data QC procedure was then separately applied to the cases (along with DFCI controls) and primary comparator controls since they come from different sources. For the cases and DFCI controls, 125 samples were excluded for high missing data rates of $<95 \%$ completion or outlier over three SD outlier proportions of heterozygous genotypes, or incorrect gender, or genotypic relatedness to other subjects (IBD $\hat{\pi}>0.185$ ) or unexpected duplicates, or reclassified ineligible diagnosis or population outliers (over $3 \mathrm{SD}$ ) of the first two principal components using HapMap III with EIGENSTRAT (Price et al. 2006). This left 888 cases and 259 DFCI controls that were genotyped. After combining these 259 DFCI controls with the 4622 NHS and HPFS healthy primary comparator controls, we had a total of 4881 controls.

Common SNPs $(646,368)$ for cases and controls were identified, and their data were merged. Similar QC measures were then performed using PLINK (Purcell et al. 2007) on the merged data to ensure homogeneity in terms of missingness and ancestry in the combined sample. Three cases and 18 controls were excluded for high missing data rates of $<95 \%$ completion, or genotypic relatedness to other subjects (IBD $\hat{\pi}>0.185$ ). We then used PLINK to select 105,845 independent markers (pairwise $\left.r^{2}<0.2\right)$ to conduct a principal component analysis to detect potential population stratification. One hundred and thirty-three outliers (53 cases and 80 controls, over six SD) of the first ten principal components were identified and removed. We then extracted SNPs with MAF $>1 \%$, genotyping call rate $>95 \%, P$ value for Hardy-Weinberg Equilibrium (HWE) test in the controls $>10^{-5}, P$ value for HWE in combined cases and controls $>10^{-6}$ (to eliminate allele flips) and $P$ value for difference in missing data rate between cases and controls $>10^{-6}$. To identify markers that were potentially genotyped with error, we performed a logistic regression comparing DFCI controls with Harvard controls and eliminated SNPs with a $P$ value smaller than 0.01 . The final data contain 613,218 SNPs from 5615 samples (832 cases and 4783 controls).

Published by Bioscientifica Ltd 
Table 1 Summary characteristics of cases and controls used in the GWAS.

\begin{tabular}{|c|c|c|c|c|c|}
\hline Cases & $\boldsymbol{N}$ & $\begin{array}{l}\text { Gender } \\
\text { (M/F) }\end{array}$ & $\begin{array}{l}\text { Median age } \\
\text { (years) }\end{array}$ & $\begin{array}{c}\text { Tumor grade } \\
\text { (well differentiated/ poorly } \\
\text { differentiated) }\end{array}$ & $\begin{array}{c}\text { Tumor stage at diagnosis } \\
\text { (localized/metastatic) }\end{array}$ \\
\hline Overall cohort & 832 & $384 / 448$ & 54.7 & $793 / 39$ & $472 / 399$ \\
\hline Small intestine NET & 293 & $139 / 154$ & 56.5 & $291 / 2$ & $134 / 159$ \\
\hline Pancreatic NET & 156 & $83 / 73$ & 53.1 & $147 / 9$ & $63 / 93$ \\
\hline Bronchial NET & 128 & $41 / 87$ & 56.3 & $124 / 4$ & $115 / 13$ \\
\hline Other NET & 255 & $121 / 134$ & 54.3 & $231 / 24$ & $121 / 134$ \\
\hline \multicolumn{6}{|l|}{ Controls } \\
\hline DFCl controls & 241 & $96 / 145$ & 55.4 & NA & NA \\
\hline Harvard controls & 4542 & $2025 / 2517$ & 60.8 & NA & NA \\
\hline
\end{tabular}

\section{Statistical analysis}

We used an additive genetic model (test for trend) to evaluate the associations of SNP with neuroendocrine tumor risk. Logistic regression was performed, comparing all cases to all controls, for each SNP, adjusting for gender, age (at diagnosis for cases and at blood draw for controls) and the first 10 principal components (PCs). We used PLINK to perform regression analyses. Measures of the genomic control lambda close to one and an inspection of a quantile-quantile plot did not demonstrate a systematic deviation from the expected distribution, minimizing the likelihood of systematic genotype error bias due to underlying population substructure (Price et al. 2006). We set the genome-wide significance threshold at $5 \times 10^{-8}$.

\section{Results}

\section{Demographics of patients and controls}

After quality control, 862 cases, 241 DFCI controls (QC-controls) and 4542 Harvard controls were used in the analysis. The median age of the cases was 54.7 years, and the median age of the Harvard controls was 60.8; a slight
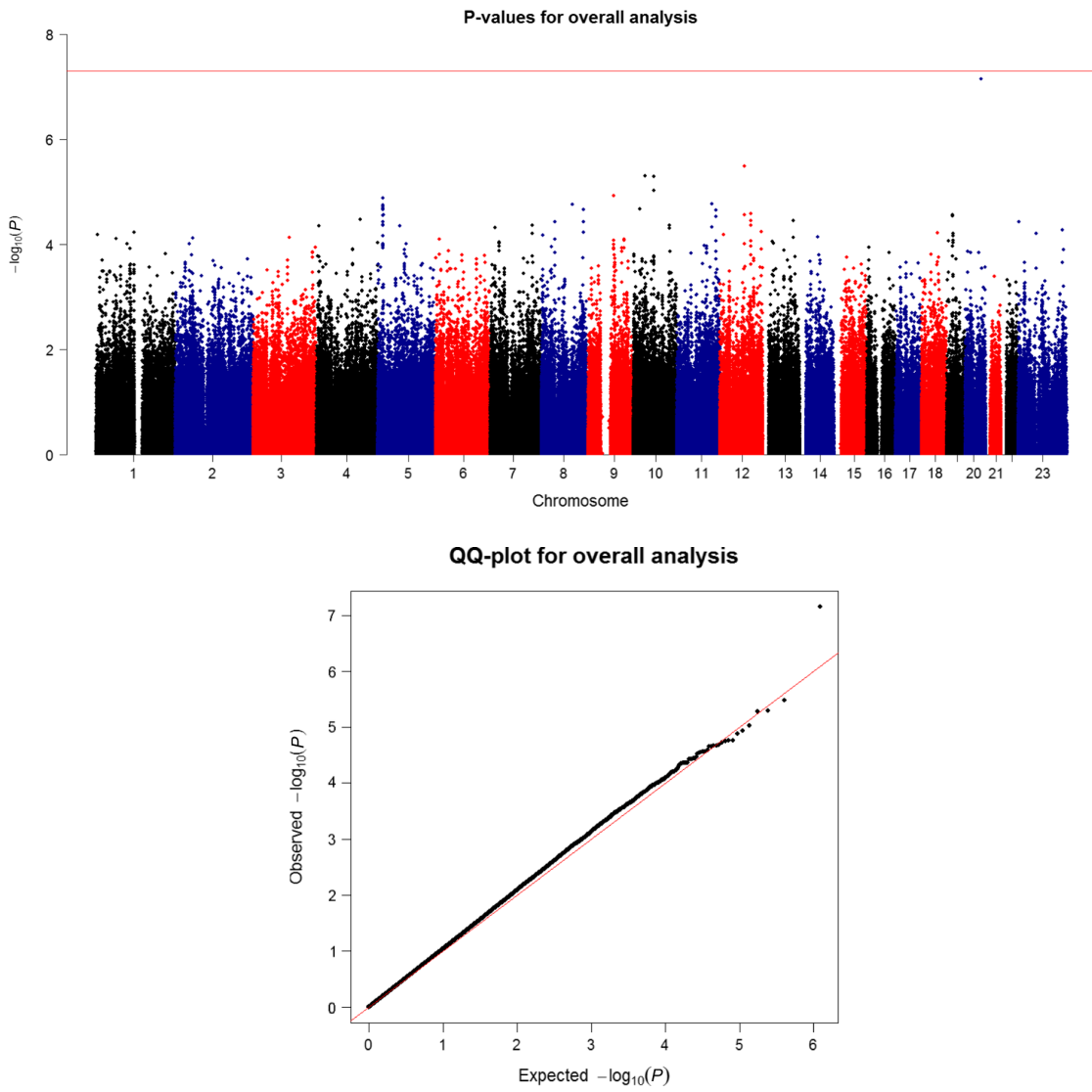

Figure 1

Manhattan and QQ plots for the overall risk analysis. Associations were obtained using logistic regression analysis with adjustment for age, sex and the top 10 principal components of population stratification. In the Manhattan plot, the $-\log _{10} P$ values (y axis) of 613,218 SNPs from 832 NET cases and 4783 controls are presented on the basis of their chromosomal positions ( $x$ axis). The red horizontal line indicates the genome-wide significance threshold $5 \times 10^{-8}$. In the QQ plot, the observed $-\log _{10} P$ values (y axis) are compared with the corresponding expected $-\log _{10} P$ values (x axis) 
Table 2 Summary of SNPs associated with risk in small intestine NET.

\begin{tabular}{|c|c|c|c|}
\hline & SNP & chr & Alleles \\
\hline \multirow{3}{*}{$\begin{array}{l}\text { Small intestine subgroup } \\
\qquad\left(N_{\text {case }}=293, N_{\text {control }}=4783\right)\end{array}$} & rs2192799 & 12 & $\mathrm{~T} / \mathrm{C}$ \\
\hline & rs2540513 & 12 & $A / G$ \\
\hline & rs256182 & 12 & $\mathrm{G} / \mathrm{T}$ \\
\hline
\end{tabular}

Based on predefined cutoff value of $P<5 \times 10^{-8}$.

female preponderance was observed in both cases and controls. Of the cases, 293 had small intestine tumors, 156 pancreatic neuroendocrine tumors and 128 bronchial neuroendocrine tumors. Only $4 \%$ of the cases had tumor histologies classified as poorly differentiated. Although complete ethnicity data on all cases were not available, of the available cases, 93\% self-reported as Caucasian. The primary controls consisted of healthy Caucasian individuals identified from the Nurses' Health Study (NHS) and the Health Professionals Follow-Up Study (HPFS). Further characteristics of the cases and controls are shown in Table 1.

\section{Overall risk analysis}

A logistic regression comparing all NET cases to the Harvard controls was first performed for each SNP, adjusting for gender, age (at diagnosis for cases and at blood draw for controls) and the first 10 PCs. No SNP passed the genome-wide significance threshold of $P<5 \times 10^{-8}$. Figure 1 displays the Manhattan plot and the quantile-quantile (QQ) plot for the overall analysis. The genomic control $\lambda$ was 1.06 .

\section{Subgroup analyses for risk}

Given potential genetic differences between lung, small intestine and pancreatic NET, we also evaluated risk associations in these subgroups separately. Genetic differences between well-differentiated and poorly differentiated tumors have also been reported, but the small number of poorly differentiated tumors in our cohort precluded this subgroup analysis. For lung NET, a logistic regression comparing 128 lung carcinoid cases to the Harvard controls was performed for each SNP, adjusting for gender, age (at diagnosis for cases and at blood draw for controls) and the first 10 PCs. The genomic control $\lambda$ was 1.12. Although one SNP, rs10827565, on chromosome 10 , passed the genome-wide significance threshold (Odds ratio $2.992, P=9.7 \mathrm{e}-12$ ), it is not located on a known gene, and we were not able to identify any SNPs in LD with this SNP to confirm the association. The potential significance

\begin{tabular}{c} 
Case MAF \\
\hline 0.61 \\
0.62 \\
0.61 \\
\hline
\end{tabular}

\begin{tabular}{c}
\hline Control MAF \\
\hline 0.49 \\
0.49 \\
0.48 \\
\hline
\end{tabular}

\begin{tabular}{c} 
Odds ratio $(95 \% \mathrm{Cl})$ \\
\hline 1.86 \\
1.87 \\
1.8
\end{tabular}

$P$ value
$\begin{aligned} & 3.7 \mathrm{e}-09 \\ & 2.8 \mathrm{e}-09 \\ & 3.6 \mathrm{e}-08\end{aligned}$

of this SNP is, therefore, not clear. We performed a parallel analysis for pancreatic NET cases, where no SNP passed genome-wide significance and no clear risk associations were identified.

For small intestine NET, we performed a logistic regression comparing 293 small bowel carcinoid cases to the controls for each SNP, adjusting for gender, age (at diagnosis for cases and at blood draw for controls) and the first 10 PCs. The genomic control $\lambda$ was 1.11 . Three SNPs in high LD with each other $\left(r^{2}>0.97\right)$, rs2192799, rs2540513 and rs256182, passed the genome-wide significance threshold (Table 2). All three SNPs are located on chromosome 12 within a $200 \mathrm{~kb}$ noncoding region between the genes LTAH and ELK3 (Fig. 2). Using the 1000 Genome phase 3 CEU data, we looked at all the bi-allelic SNPs within $500 \mathrm{~kb}$ of the three SNPs of interest for SNPs

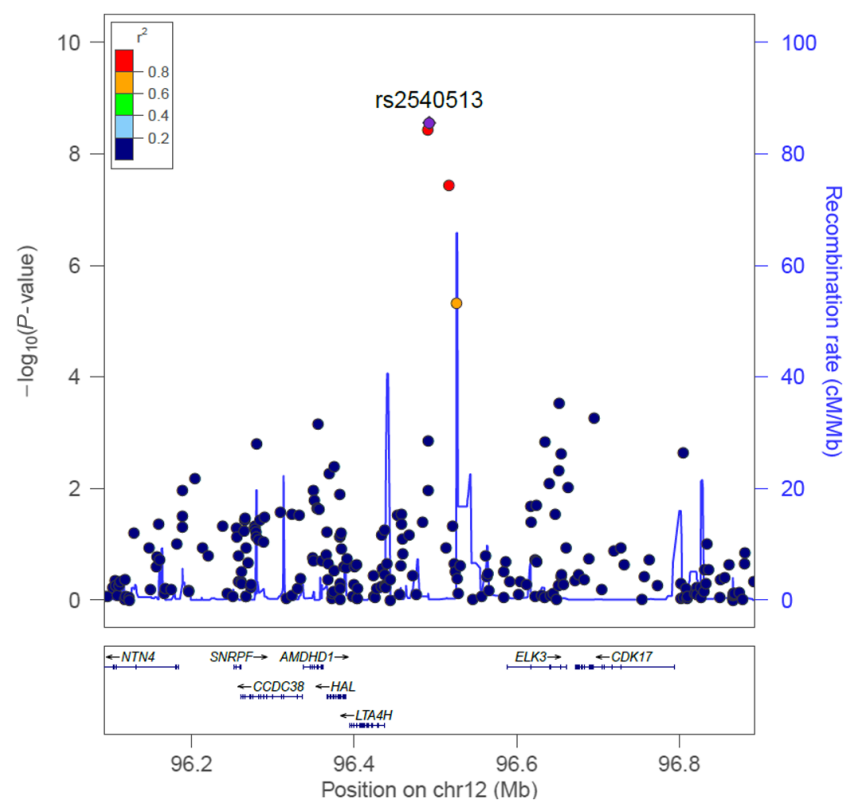

Figure 2

Small intestine NET association plot for $\pm 400 \mathrm{~kb}$ region around $\mathrm{rs} 2540513$ on chromosome 12. The $-\log _{10} P$ values (y axis) of the SNPs are presented according to their chromosomal positions (x axis). The genetic recombination rates (cM/Mb) estimated using the 1000 Genomes November 2014 EUR samples are shown with a blue line. Genes within this region were annotated and shown as arrows. The $r^{2}$ values of the SNPs with top SNP rs2540513 are indicated by different colors.

Published by Bioscientifica Ltd. 
that were in LD with the three identified SNPs. Out of a total of 29,307 bi-allelic SNPs in this region, we found 16 SNPs that were in high LD $\left(r^{2}>0.8\right)$ with our three SNPs, all within a $30 \mathrm{~kb}$ region (the following SNPs were identified: rs2192799, rs2540513, rs2660861, rs2660859, rs4762270, rs2660858, rs2660855, rs4762135, rs256189, rs256182, rs2098357, rs42436, rs34576, rs34577, rs34578, rs34579). None, however, were on a known gene. We further tested correlations between these three SNPs and 14 genes located within one $\mathrm{MB}$ using the Genevar database (Yang et al. 2010). This analysis revealed a potential association between one of the SNPs, rs256182, and expression of ELK3, a transcription factor implicated in angiogenesis (correlation $0.255, P=0.0076$ ), in lymphoblastoid cells.

\section{Discussion}

Genetic risk factors for neuroendocrine tumors remain poorly defined. Although Mendelian syndromes associated with specific inherited mutations have been associated with the development of neuroendocrine tumors, such mutations account for only a minority of cases and the great majority of neuroendocrine tumors are thought to be sporadic. The relative rarity of neuroendocrine tumors has been an obstacle to performing large, genomewide association studies to identify genetic risk factors. Our study, which comprised over 800 cases and over 4500 controls, represents one of the largest such studies performed to date.

We did not identify any risk associations in our cohort overall, nor did we identify risk associations in the subgroup of cases with pancreatic neuroendocrine tumors. The identification of only a single associated SNP in lung neuroendocrine tumors not located in any known gene and without other associated confirming SNPs suggests this finding may be spurious and should be interpreted with caution. Our analysis of the small intestine neuroendocrine tumor subgroup, on the other hand, revealed three associated SNPs, all in strong linkage disequilibrium on chromosome 12q23. These SNPs are not located on any known gene, though they are in close proximity to ELK3 (Net), a gene implicated in transcriptional regulation of angiogenesis. Evaluation of the Genevar database suggested that these SNPs are potentially associated with the expression of ELK3, albeit in a different (lymphoblastoid) cell type. In mouse studies, ELK3 is expressed at sites of angiogenesis during early development; both phosphorylated ELK3 and VEGF are coexpressed in human tumors (Zheng et al. 2003). The finding of a potential association with angiogenesis is particularly intriguing in light of the fact that neuroendocrine tumors are characterized by abundant vasculature, and are responsive to inhibitors of the VEGF pathway (Kulke et al. 2008b). Von HippelLindau disease, one of the inherited cancer syndromes associated with the development of neuroendocrine tumors, is caused by germline mutations in VHL. VHL functions, in part, by regulating the degradation of HIF1A (hypoxia inducible factor 1A), a transcription factor that induces the expression of a number of genes implicated in angiogenesis (Richard et al. 2013). Mutations in EPAS1 (hypoxia inducible factor 2A), a homologous gene transcription factor also implicated in angiogenesis, have been reported in pheochromocytoma (Zhuang et al. 2012, Comino-Mendez et al. 2013). The three SNPs identified on chromosome $12 \mathrm{q} 23$ are also in proximity to two other genes, LTAH4 and HAL, though whether these genes could be implicated in the development of neuroendocrine tumors is not clear. LTAH4 is a gene encoding leukotriene A4 hydrolase, an amino acid peptidase involved in arachidonic acid metabolism, and has been linked to the development of emphysema (Paige et al. 2014). $H A L$ encodes histidine ammonia lyase, a gene associated with histidinemia (Kawai et al. 2005).

The identification of germline risk associations limited to the small intestine subgroup is in some ways not surprising in light of the fact that it has become increasingly apparent that somatic genetic changes in neuroendocrine tumors vary significantly according to neuroendocrine tumor subtype. Somatic mutations in neuroendocrine tumors are now well characterized and differ significantly depending upon tumor site of origin. In addition to mutations in MEN1, pancreatic neuroendocrine tumors have been reported to commonly contain recurrent mutations in two other genes, $D A X X$ and $A T R X$, both implicated in chromosomal maintenance (Jiao et al. 2011). Additional mutations in genes implicated in the mTOR pathway, including TSC2 and PTEN, were reported in 15\% of cases in this study. Recently, recurrent mutations in the transcription factor YY1 (Yin Yang 1), a gene implicated in mitochondrial function and insulin/insulin-like growth factor signaling, were identified in a series of 10 insulinomas (Cao et al. 2013). Although mutations in MEN1 have been identified in bronchial carcinoid tumors, mutations in DAXX, ATRX and mTOR pathway genes have not (Fernandez-Cuesta et al. 2014). Somatic mutations in small intestine neuroendocrine tumors are quite uncommon; recurrent mutations in $C D K N 1 B$ (p27) have been reported, but were present in only $8 \%$

Published by Bioscientifica Ltd 
of cases evaluated using a combination of whole genome and targeted sequencing (Francis et al. 2013). Both familial and sporadic small intestine neuroendocrine tumors have been characterized by deletions and loss of chromosome 18 , though a specific gene candidate at this location has not been identified (Kulke et al. 2008a, Cunningham et al. 2011).

Our results differ from those of two previous studies evaluating genetic risk factors in neuroendocrine tumors. The first of these studies, a pilot genome-wide association evaluated potential risk associations in a cohort of 239 small intestine neuroendocrine tumors, including 82 cases from our center, and 110 controls. This study utilized an earlier Illumina $300 \mathrm{~K}$ chip and identified potential associations with a variant in the gene KIF16B, a kinesin-like protein located on chromosome 20p12, that approached though did not reach statistical significance. This study also identified a potential association between copy number variation in chromosome 18 and the development of small intestine neuroendocrine tumors (Walsh et al. 2011). We did not confirm an association with KIF16B in our study, and were not able to evaluate copy number variation in our dataset to assess this potential association. A second study, performed at our center, focused on a limited number of SNPs in 355 candidate genes, using a discovery set of 261 cases and 319 controls and a replication set of 235 additional cases and 113 controls (Ter-Minassian et al. 2011). This study reported modest risk associations associated with single SNPS in IL12A and DAD1. We did not confirm these associations in the current study, which not only included a significantly expanded number of cases, but also utilized a new, larger and independent group of controls.

Though significantly larger than previous studies in neuroendocrine tumors, our study is still smaller than genome-wide association studies reported in other malignancies and is limited in its power to detect associations. Additionally, in light of the increasing evidence that neuroendocrine tumors are genetically heterogeneous, genetic predisposing factors likely vary across tumor subtypes. Although we attempted to address this potential weakness by performing subgroup analyses, these analyses are based on an even more limited number of cases, resulting in the potential to both miss associations due to the lack of statistical power, as well as the potential to identify spurious associations.

In summary, our genome-wide analysis identified a potential genetic risk locus on 12q23 associated with small intestine neuroendocrine tumor risk. This locus is in proximity to $E L K 3$, a transcription factor implicated in angiogenesis. Our results provide a basis for initial exploration of the role of genes associated with this locus, as well as replication studies to confirm the observed associations. Additional and larger studies to evaluate potential genetic risk factors for sporadic pancreatic and bronchial neuroendocrine tumors are warranted.

\section{Declaration of interest}

The authors declare that there is no conflict of interest that could be perceived as prejudicing the impartiality of the research reported.

\section{Funding}

This work was supported by Grant \#R01CA151532 (to M H K), Dana-Farber/Harvard SPORE Grant in Gastrointestinal Center (Grant \# P50CA127003) and Goldhirsh-Yellin Foundation Fund for Neuroendocrine Tumor Research. The authors gratefully acknowledge additional support from the Gitta and Saul Kurlat Fund for Neuroendocrine Tumor Research, Jane Dybowski Fund for Neuroendocrine Cancer, McIntyre Family Fund for Neuroendocrine Tumor Research, Lipson Family Fund, the Murphy Family Fund for Carcinoid Tumor Research, and the Caring for Carcinoid Foundation.

\section{References}

Babovic-Vuksanovic D, Constantinou CL, Rubin J, Rowland CM, Schaid DJ \& Karnes PS 1999 Familial occurrence of carcinoid tumors and association with other malignant neoplasms. Cancer Epidemiology Biomarkers and Prevention 8 715-719.

Cao Y, Gao Z, Li L, Jiang X, Shan A, Cai J, Peng Y, Li Y, Huang X, Wang J, et al. 2013 Whole exome sequencing of insulinoma reveals recurrent T372R mutations in YY1. Nature Communications 4 2810. (doi:10.1038/ncomms3810)

Comino-Mendez I, de Cubas AA, Bernal C, Alvarez-Escola C, Sanchez-Malo C, Ramirez-Tortosa CL, Pedrinaci S, Rapizzi E, Ercolino T, Bernini G, et al. 2013 Tumoral EPAS1 (HIF2A) mutations explain sporadic pheochromocytoma and paraganglioma in the absence of erythrocytosis. Human Molecular Genetics 22 2169-2176. (doi:10.1093/hmg/ddt069)

Cunningham JL, Diaz de Stahl T, Sjoblom T, Westin G, Dumanski JP \& Janson ET 2011 Common pathogenetic mechanism involving human chromosome 18 in familial and sporadic ileal carcinoid tumors. Genes Chromosomes \& Cancer 50 82-94. (doi:10.1002/ gcc.20834)

Fernandez-Cuesta L, Peifer M, Lu X, Sun R, Ozretic L, Seidel D, Zander T, Leenders F, George J, Muller C, et al. 2014 Frequent mutations in chromatin-remodelling genes in pulmonary carcinoids. Nature Communications 5 3518. (doi:10.1038/ncomms4518)

Fishbein L 2016 Pheochromocytoma and paraganglioma: genetics, diagnosis, and treatment. Hematology/Oncology Clinics of North America 30 135-150. (doi:10.1016/j.hoc.2015.09.006)

Francis JM, Kiezun A, Ramos AH, Serra S, Pedamallu CS, Qian ZR, Banck MS, Kanwar R, Kulkarni AA, Karpathakis A, et al. 2013 Somatic mutation of CDKN1B in small intestine neuroendocrine tumors. Nature Genetics 45 1483-1486. (doi:10.1038/ng.2821)

Hassan MM, Phan A, Li D, Dagohoy CG, Leary C \& Yao JC 2008a Family history of cancer and associated risk of developing neuroendocrine tumors: a case-control study. Cancer Epidemiology, Biomarkers and Prevention 17 959-965. (doi:10.1158/ 1055-9965.epi-07-0750) 
Hassan MM, Phan A, Li D, Dagohoy CG, Leary C \& Yao JC 2008b Risk factors associated with neuroendocrine tumors: a U.S.-based case-control study. International Journal of Cancer 123 867-873. (doi:10.1002/ijc.23529)

Hemminki K \& Li X 2001 Incidence trends and risk factors of carcinoid tumors: a nationwide epidemiologic study from Sweden. Cancer 92 2204-2210. (doi:10.1002/1097-0142(20011015)92:8<2204::aidcncr1564>3.0.co;2-r)

Hiripi E, Bermejo JL, Sundquist J \& Hemminki K 2009 Familial gastrointestinal carcinoid tumours and associated cancers. Annals of Oncology 20 950-954. (doi:10.1093/annonc/mdn706)

Jiao Y, Shi C, Edil BH, de Wilde RF, Klimstra DS, Maitra A, Schulick RD, Tang LH, Wolfgang CL, Choti MA, et al. 2011 DAXX/ATRX, MEN1, and mTOR pathway genes are frequently altered in pancreatic neuroendocrine tumors. Science 331 1199-1203. (doi:10.1126/ science.1200609)

Kawai Y, Moriyama A, Asai K, Coleman-Campbell CM, Sumi S, Morishita H \& Suchi M 2005 Molecular characterization of histidinemia: identification of four missense mutations in the histidase gene. Human Genetics 116 340-346. (doi:10.1007/ s00439-004-1232-5)

Kharazmi E, Pukkala E, Sundquist K \& Hemminki K 2013 Familial risk of small intestinal carcinoid and adenocarcinoma. Clinical Gastroenterology and Hepatology 11 944-949. (doi:10.1016/ j.cgh.2013.02.025)

Korn JM, Kuruvilla FG, McCarroll SA, Wysoker A, Nemesh J, Cawley S, Hubbell E, Veitch J, Collins PJ, Darvishi K, et al. 2008 Integrated genotype calling and association analysis of SNPs, common copy number polymorphisms and rare CNVs. Nature Genetics $\mathbf{4 0}$ 1253-1260. (doi:10.1038/ng.237)

Kulke MH 2007 Clinical presentation and management of carcinoid tumors. Hematology/Oncology Clinics of North America 21 433-455. (doi:10.1016/j.hoc.2007.04.004)

Kulke M \& Mayer R 1999 Carcinoid tumors. New England Journal of Medicine 340 858-868. (doi:10.1056/NEJM199903183401107)

Kulke MH, Freed E, Chiang DY, Philips J, Zahrieh D, Glickman JN \& Shivdasani RA 2008a High-resolution analysis of genetic alterations in small bowel carcinoid tumors reveals areas of recurrent amplification and loss. Genes Chromosomes \& Cancer 47 591-603. (doi:10.1002/gcc.20561)

Kulke MH, Lenz HJ, Meropol NJ, Posey J, Ryan DP, Picus J, Bergsland E, Stuart K, Tye L, Huang X, et al. 2008b Activity of sunitinib in patients with advanced neuroendocrine tumors. Journal of Clinical Oncology 26 3403-3410. (doi:10.1200/ jco.2007.15.9020)

Leotlela PD, Jauch A, Holtgreve-Grez H \& Thakker RV 2003 Genetics of neuroendocrine and carcinoid tumours. Endocrine-Related Cancer 10 437-450. (doi:10.1677/erc.0.0100437)

Paige M, Wang K, Burdick M, Park S, Cha J, Jeffery E, Sherman N \& Shim YM 2014 Role of leukotriene A4 hydrolase aminopeptidase in the pathogenesis of emphysema. Journal of Immunology 192 5059-5068. (doi:10.4049/jimmunol.1400452)

Price AL, Patterson NJ, Plenge RM, Weinblatt ME, Shadick NA \& Reich D 2006 Principal components analysis corrects for stratification in genome-wide association studies. Nature Genetics 38 904-909. (doi:10.1038/ng1847)
Purcell S, Neale B, Todd-Brown K, Thomas L, Ferreira MA, Bender D, Maller J, Sklar P, de Bakker PI, Daly MJ, et al. 2007 PLINK: a tool set for whole-genome association and population-based linkage analyses. American Journal of Human Genetics $\mathbf{8 1}$ 559-575. (doi:10.1086/519795)

Richard S, Gardie B, Couve S \& Gad S 2013 Von Hippel-Lindau: how a rare disease illuminates cancer biology. Seminars in Cancer Biology 23 26-37. (doi:10.1016/j.semcancer.2012.05.005)

Rinzivillo M, Capurso G, Campana D, Fazio N, Panzuto F, Spada F, Cicchese N, Partelli S, Tomassetti P, Falconi M, et al. 2015 Risk and protective factors for small intestine neuroendocrine tumours: a prospectivecase-control study. Neuroendocrinology 103 516-522. (doi:10.1159/000440884)

Sei Y, Zhao X, Forbes J, Szymczak S, Li Q, Trivedi A, Voellinger M, Joy G, Feng J, Whatley M, et al. 2015 A hereditary form of small intestinal carcinoid associated with a germline mutation in inositol polyphosphate multikinase. Gastroenterology 149 67-78. (doi:10.1053/j.gastro.2015.04.008)

Sei Y, Feng J, Zhao X, Forbes J, Tang D, Nagashima K, Hanson J, Quezado MM, Hughes MS \& Wank SA 2016 Polyclonal crypt genesis and development of familial small intestinal neuroendocrine tumors. Gastroenterology 151 140-151. (doi:10.1159/000440884)

Ter-Minassian M, Wang Z, Asomaning K, Wu MC, Liu CY, Paulus JK, Liu G, Bradbury PA, Zhai R, Su L, et al. 2011 Genetic associations with sporadic neuroendocrine tumor risk. Carcinogenesis 32 1216-1222. (doi:10.1093/carcin/bgr095)

Thevenon J, Bourredjem A, Faivre L, Cardot-Bauters C, Calender A, Le Bras M, Giraud S, Niccoli P, Odou MF, Borson-Chazot F, et al. 2015 Unraveling the intrafamilial correlations and heritability of tumor types in MEN1: a Groupe d'etude des Tumeurs Endocrines study. European Journal of Endocrinology 173 819-826. (doi:10.1530/ EJE-15-0691)

Walsh KM, Choi M, Oberg K, Kulke MH, Yao JC, Wu C, Jurkiewicz M, Hsu LI, Hooshmand SM, Hassan M, et al. 2011 A pilot genome-wide association study shows genomic variants enriched in the non-tumor cells of patients with well-differentiated neuroendocrine tumors of the ileum. Endocrine-Related Cancer 18 171-180. (doi:10.1677/ERC-10-0248)

Yang TP, Beazley C, Montgomery SB, Dimas AS, Gutierrez-Arcelus M, Stranger BE, Deloukas P \& Dermitzakis ET 2010 Genevar: a database and Java application for the analysis and visualization of SNP-gene associations in eQTL studies. Bioinformatics 26 2474-2476 (doi:10.1093/bioinformatics/btq452).

Yao JC, Hassan M, Phan A, Dagohoy C, Leary C, Mares JE, Abdalla EK, Fleming JB, Vauthey JN, Rashid A, et al. 2008 One hundred years after "carcinoid": epidemiology of and prognostic factors for neuroendocrine tumors in 35,825 cases in the United States. Journal of Clinical Oncology 26 3063-3072. (doi:10.1200/ JCO.2007.15.4377)

Zheng H, Wasylyk C, Ayadi A, Abecassis J, Schalken JA, Rogatsch H, Wernert N, Maira SM, Multon MC \& Wasylyk B 2003 The transcription factor Net regulates the angiogenic switch. Genes and Development 17 2283-2297. (doi:10.1101/gad.272503)

Zhuang Z, Yang C, Lorenzo F, Merino M, Fojo T, Kebebew E, Popovic V, Stratakis CA, Prchal JT \& Pacak K 2012 Somatic HIF2A gain-of-function mutations in paraganglioma with polycythemia. New England Journal of Medicine 367 922-930. (doi:10.1056/ NEJMoa1205119)

Received in final form 14 May 2016 Accepted 20 June 2016
() 2016 Society for Endocrinology Printed in Great Britain
Published by Bioscientifica Ltd. 